\title{
SUR LA DÉFINITION DES SECTIONS EFFICACES
}

\author{
Par JACQUes WINTER.
}

\begin{abstract}
Sommaire. - On étudie le sens physique qu'on peut donner aux sections efficaces, et notamment, ce que signifie le fait qu'elles peuvent devenir infinies, pour des ondes incidentes de plus en plus lentes Ceci nous permet de donner une théorie simple de la diffaction des pinceaux, sans faire intervenir explicitement le principe d'Huygens, mais seulement les propriétés, données ailleurs, du développement de Rayleigh.
\end{abstract}

1. Définition des sections efficaces. - Nous ne reprenons pas ici la théorie de la diffusion élastique d'urie onde plane électronique de de Broglie $\mathrm{e}^{-i K r}$ par une zone de potentiel sphérique ('). Nous supposons connues les définitions des déphasages des divers ordres $\delta_{n}$ et les propriétés de ces déphasages. Nous rappellerons simplement la définition des sections efficaces des divers ordres $Q_{n}$.

On considère l'onde diffusée d'ordre $n$.

$\Psi_{n}^{(d)}=c_{n}(-i)^{n} \sqrt{\frac{\pi}{2 K r}}(2 n+1) H_{n+\frac{1}{2}}^{(2)}(K r) P_{n}(\cos \theta)$

avec

$$
\left|c_{n}\right|=\sin \delta_{n} .
$$

Aux très grandes distances on a

$$
H_{n+\frac{1}{2}}^{(\mathscr{x})}=\sqrt{\frac{2}{\pi K r}} \mathrm{e}^{-i\left(k r-\frac{n+1}{\sharp} \pi\right)} .
$$

Le flux diffusé normalement à travers une sphère de très grand rayon vaut: Partie Imaginaire de $\Psi \frac{\partial \Psi^{\circ}}{\partial r}$, et en intégrant sur toute la sphère :

$$
4 \pi \sin ^{2} \delta_{n} \frac{(2 n+1)}{K} .
$$

(Les phénomènes d'interférence de l'onde incidente et de l'onde diffusée ne modifient pas le résultat de ce calcul simple.)

Exprimons ce flux en prenant pour unité le flux envoyé par l'onde incidente à travers l'unité de surface, qui vaut $K\left({ }^{2}\right)$. On aura

$$
4 \pi \sin ^{2} \widehat{\theta}_{n} \frac{(2 n+1)}{\Lambda^{2}},
$$

(1) Faxey et Holtsmarck. " Physik, 1997, 45, p. 307.

Alus et Morse. $Z$. 'Mhysih. 1931 , t. 70 , p. 567.

L de Broglie. Ann. Inst. Poincare, 1933, t. 3 , p. 349

Yous conservons les notations usuelles, et adoptons les unités de Hartree : $e=m=1, h=2 \pi$.

() Dans notre thèse (Annales de Physique, 1934, s. 11, t. 2 , p. '́x̆ŏ, nous avons oublié de reproduire cette définition, ce qui rend difficile à comprendre l'expression des sections efficaces, que nous appelons, à tort, flux. Cela n'entraine pas de efficaces, que nous appelons, à tort, flux. Cela n'entraine pas de
conséquences, car nous n'étudions que la répartition angulaire des flux diffusés et non l'effet Ramsauer, ou leur variation arec $K$. expression qui a les dimensions d'une surface, et qui caractérise la perte par diffusion subie par le flux incident traversant l'unité de surface. C'est la section efficace d'indice $n, Q_{n}$.

\section{Difficulté due aux sections efficaces infinies.} - Supposons que $K$ tende vers zéro (électrons très. lents) et supposons que $\sin \delta_{n}$ qui est une fonction de $K$, ne tende pas vers zéro, mais vers une limite $\alpha_{0}\left({ }^{1}\right)$.

Dirigeons sur le corpuscule diffuseur un large faisceau cylindrique, de section $S$. Le rapport du flux diffusé au flux incident sera un nombre qui vaudra

$$
4 \pi \sin ^{2} \delta_{n} \frac{(2 n+1)}{S K^{2}}
$$

el lorsque $K$ tendra vers zéro, il tendra vers l'infini. Autrement dit, il y aurait infiniment plus d'électrons. diffusés que d'électrons incidents.

3. Propriétés du pinceau incident. - Pour élucider cette difficulté. il est nécessaire de partir du développement de Rayleigh.

$\mathrm{e}^{-i \hbar x}=\sum_{n=0}^{\infty}(-\imath)^{n} \sqrt{\frac{\pi}{2 K r}}(2 n+1) \cdot J_{n+\frac{1}{2}}(K r) P_{n}(\cos \theta)(\check{\zeta})$

et d'arrêter à l'indice $N$ le développement du deuxième membre. Ce deuxième membre représente alors un pin-

(1) Cherchons à obtenir ce résultat; supposons le potentiel diffuseur representé par une zone sphérique de rayon $R$, et de potentiel constant: on a alors (voir L. DE Broglie. Loc. cit.) en posant $K_{1}=$ quantité de mouvement de l'onde de de Broglie dans la sphère

$$
c_{0}=\mathrm{e}^{k R} \frac{K \sin \dot{k}_{1} R \cos K R-K_{1} \sin K R \cos K_{1} R}{\imath K_{1} \cos K_{1} k-k \sin K_{1} k} .
$$

Supposons que $K$ tende vers zéro, $K$ tendra vers une limite fixe, qui ne dépend que du potentiel dans la sphère. Le numérateur de $c_{0}$ devient équivalent à

$$
K\left[\sin K_{1} R-K_{1} R \cos K_{1} R\right]
$$

$K$ étant très pelit, mais fixé, nous pouvons déterminer $K_{1}$; arbitrairement grand de facon que $\cos K_{1} R=0$. Le dénominateur prendra son plus petit module possible, soit $K \sin K_{1} R$ et $c_{0}$-devient égal à 1 .

En s'écartant légèrement de la valeur précédente de $K_{1}$. on aura pour $c_{i}$ telle valeur qu'on voud ra (de module inférieur à 1 ), pour $K$ arbitrairement petit. 


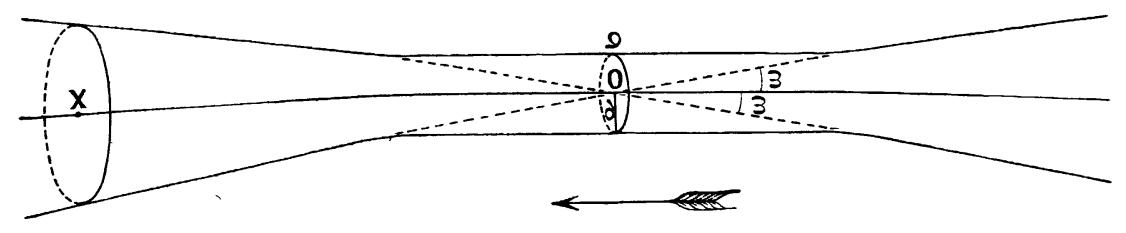

Fig. 1.

ceau, dont nous avons étudié les propriétés. Rappelons les résultats essentiels obtenus ${ }^{(1)}$.

a) Le pinceau peut ètre grossièrement représenté par un cylindre, parallèle à la direction de propagation, mais qui s'ouvre aux extrémités comme l'indique la figure, avec une ouverture conique $2 \varepsilon$, qui ne dépend que de $N$. L'ordre de grandeur de $\varepsilon$ vaut

$$
\varepsilon \sim \frac{1}{N} \text {. }
$$

b) Il transporte un flux qui vaut sensiblement $\%, \lambda^{r_{2}} / \boldsymbol{K}$, $\gamma$ étant un coefficient numérique indépendant de $N$ et de $K$ ( $N$ est très grand par hypothèse).

Appelons $p$ le rayon de la section centrale $\sigma$ du pinceau. Nous admettrons que l'on peut poser

$$
\rho=\sqrt{\frac{\gamma}{\pi}} \frac{N}{K}
$$

ce qui se justifie ainsi :

$1^{\circ} \mathrm{Au}$ centre du pinceau, le flux par unité de surface vaut $K$, puisqu'en cette région le pinceau est très exactement représenté par l'expression $\mathbf{e}^{-i K x}$. Il est naturel d'étendre cette propriété à la totalité de la section du pinceau, puisque le pinceau représente sensiblement un flux plan homogène, en sa région centrale, et puisqu'on élimine les zones périphériques qu ne véhiculent qu'une fraction négligeable du flux, et qui sont les régions où le flux s'affaiblit. D’après la propriété $b$, l'expression (7) en découle nécessairement.

20 Par raison d’homogénéité, si on peut définir un rayon $p$ il est certainement proportionnel à $1 / K$, puisque le deuxième membre de (5) ne contient que l'argument $K r$.

Nous voyons que si $K$ croît, $N$ étant fixe, le pinceau s'étrangle et devient plus intense. A l'infini sa structure n'est pas modifiée, mais les flux diffusés décroissent proportionnellement à $1 / K$

4. Macrostruture et microstructure des flux.Nous pouvons à l'aide de ces hypothèses lever facilement la difficulté énoncée plus haut. Nous n'avons pas le droit de considérer un flux cylindrique de section $S$, mais bien un pinceau tel que ceux qu'on vient de définir. Un tel pinceau a une structure à l'infini ou macrostructure, ou une structure dans sa section $\sigma$ ou micro-

(1) Thèse, chapitre in. Ces résultats sont justifiés mais non démontrés rigoureusement. Il faut y remplacer $1 K^{2}$ par $1 / \boldsymbol{K}$ pour la raison indiquée au paragraphe 1 de cet article. structure. A l'infini le paramètre $K r$ est très grand dans $\sigma$ il est très petit. Toute la différence est là.

La définition des sections efficaces compare le flux diffusé défini macroscopiquement, au flux incident $\mathrm{e}^{-i K x}$ expression valable dans s seulement, par conséquent à un flux microscopique. On peut, pour éviter cela comparer le flux diffusé macroscopique, au flux incident macroscopique. Leur rapport est une grandeur numèrique

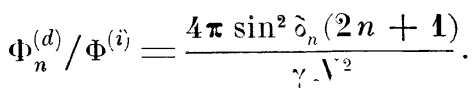

Elle ne dépend de $K$ que par l'argument $\delta_{n}$ et ne peut pas devenir infinie.

5. Comparaison des diffusions pour des valeurs différentes de $\mathbf{K}$. - La première des choses est de définir les pinceaux incidents qu'on désire comparer et qui sont caractérisés par les paramètres $N, K$ et un coefficient d'amplitude $a$ qui reste arbitraire et qu'on prendra égal à 1 pour un des 2 pinceaux. On aura donc à définir $N^{\prime}$ et $a^{\prime}$, connaissant $N, K, a=1$ et $K^{\prime}$.

a) On veut que les pinceaux aient mêmes macroflux. - Il faut prendre alors $\Lambda^{\prime}=N^{\prime}$, il en découlera $\varepsilon=\varepsilon^{\prime}$. Comme les macroflux sont divisés par $K$, il faudra prendre

$$
a^{\prime}=\sqrt{\frac{K^{\prime}}{K}} .
$$

Les macroflux diffusés seront alors dans le rapport

$$
\Phi_{n}^{(d)}\left(K^{\prime}\right) / \Phi_{n}^{(d)}(K)=\sin ^{2} \delta_{n}\left(K^{\prime}\right) / \sin ^{2} \delta_{n}(K)
$$

Si $K^{\prime}$ tend vers zéro, ce rapport tend vers $\alpha_{o}^{2} / \sin ^{2} \delta_{n}(K)$.

Il n'y a aucune difficulté pour l'interprétation de l'effet Ramsauer, et il ne surgit pas de difficulté particulière si $K^{\prime}$ tend vers zéro.

b) On veut que les pinceaux aient mêmes microflux. - Pour que les microflux aient même intensité, il faut prendre

$$
a^{\prime}=\sqrt{\frac{\pi}{\kappa^{\prime}}} .
$$

Enfin pour qu'ils aient mêmes rayons, il faut prendre

$$
N^{\prime \prime}=N \times \frac{K^{\prime \prime}}{\Lambda} \text {. }
$$

De (6), (11) et (12) il résulte que les macroflux ont 
mème valeur totale, mais non plus mème valeur en fonction des diverses valeurs de l'angle $\theta$.

On a notamment

$$
\varepsilon^{\prime}=\varepsilon \cdot \frac{K}{K^{\prime}}
$$

On aura alors, en vertu de (3) et (11)

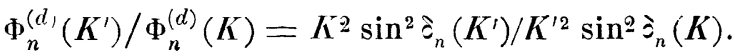

Si $K^{\prime}$ tend vers zéro, et $\operatorname{si}^{\sin ^{2} \delta_{n}}$ tend vers $\alpha_{0}$ ce rapport devient infini comme $1 / K^{2}$

La diffusion augmente indéfiniment comme la section efficace.

Quel sens physique faut-il attacher à ce résultat? Y a-t-il augmentation réelle de la diffusion? A quoi tient-elle ? Il est facile de répondre à ces questions gràce aux formules (6), (12) et (13). Lorsque $K^{\prime}$ tend vers zéro, sans qu'il y ait modification des microflux, le pinceau s'ouvre de plus en plus et aux grandes distances, perd la forme d'un pinceau cylindrique, pour prendre, dans le cas extrême, celle d'une onde sphérique convergente. Bien entendu, lorsque $\boldsymbol{K}^{\prime}$ est suffisamment petit, $N$ sera de l'ordre de quelques unités et l'on n'a un pinceau que si $N$ est très grand. Les formules posées au paragraphe 3 perdent toute signification. Néanmoins, la discussion précédente donne le sens physique des sections efficaces qui sont liées à des pinceaux d'ouvertures variables, (mais qu'on suppose négligeables) et lorsque $\boldsymbol{K}^{\prime}$ tend vers zéro, la diffusion augmente (si $x_{0}=0$ ) parce qu'on vise, en quelque sorte, la zone de ditfusion à l'aide des flux incidents.
6. Conclusion. - Il n'existe pas, à notre connais sance de définition satisfaisante des coefficients de diffusion théoriques. Une telle définition devrait tenir compte de la forme du pinceau expérimental, c'est-àdire des diaphragmes, du nombre des atomes diffuseurs traversés par le pinceau et de leur position. Une fois un pinceau formé, il n'y a pas de raisons de supposer que son ouverture angulaire varie avec $K$. Ceci suppose $N$ fixe et nous amène à la conclusion suivante: Les sections efficaces théoriques n'ont pas de sens physique précis, dans le cas limite des énergies faibles.

En effet, aux énergies suffisamment fortes et pour des diaphragmes suffisamment larges, $\varepsilon$ reste toujours très petit et négligeable, et l'emploi des sections efficaces ne soulève pas d'objection sérieuse. Il est évident qu'alors, il n'y a pas de différence réelle entre une définition des flux à l'infini, ou sur la section centrale. Ceci est clair ; mais il n'en existe pas moins un phénomène de diffraction ou de variation d'ouverture, mème lorsque $\varepsilon$ est négligıable, auquel est liée la présence des dénominateurs $K$. Si l'on peut n'en pas tenir compte dans la plupart des expériences de diffusion, c'est qu'on prend toujours $K$ assez grand. C'est cetle théorie simple de la diffraction que nous voulions donner; elle ne fait pas intervenir le principe d'Huygens. Elle ne repose que sur les propriétés intrinsèques de certains types de pinceaux, définis dans un espace sans obstacle. La définition de la diffusion peut donc se présenter de deux manières différentes, selon qu’on a à faire à des pinceaux avec ou sans ouverture angulaire sensible : Ceci r.⿲ppalle les formules élémentaires de la clarté de la lunette astronomique. 